\title{
Disturbance of respiratory muscle control in a patient with early-stage multiple sclerosis
}

\section{To the Editor:}

Respiratory dysfunction from multiple sclerosis (MS) is most commonly of insidious onset and related to progressive respiratory muscle weakness [1]. Disorders of respiratory muscle coordination are poorly recognised and challenging to diagnose. We present a case of a patient with MS and no clear muscle weakness whose dyspnoea was associated with a disorder of respiratory muscle control. The potential utility of oesophageal manometry for diagnosis of unexplained dyspnoea is highlighted.

A 54-year-old female with relapsing-remitting MS presented with 2 weeks of dyspnoea and orthopnoea. She was ambulatory with a low extended disability score of 1.5 .

6 months earlier she started cyclophosphamide for disease progression recognised by magnetic resonance imaging (MRI). A week after her last infusion she developed severe dyspnoea and orthopnoea. She had no other symptoms. Her vital signs were normal. Her laboratory tests and arterial blood gas were unremarkable. Chest computed tomography showed no pulmonary parenchymal disease. Echocardiography was unremarkable. Pulmonary function testing (PFTs) demonstrated severe restriction, with a $14 \%$ lower vital capacity in the supine position.

Her dyspnoea and PFTs improved over the following 1-2 months, but she did not return to her baseline level of pulmonary function. Electromyography (EMG) with phrenic nerve conduction studies demonstrated no abnormalities. MRI was prohibited by her orthopnoea. Previously, MRI demonstrated extensive demyelination in the brain and brainstem that was asymptomatic.

Oesophageal manometry was performed to exclude the possibility of bilateral diaphragmatic dysfunction and to elucidate her unexplained dyspnoea. A balloon-catheter was inserted via the nose to position the balloon in the distal oesophagus to estimate pleural pressure. Repeated attempts to perform a forced vital capacity manoeuvre consistently demonstrated fluctuating exhalations, as evidenced by the saw tooth pattern of the flow volume curve (fig. 1a). The simultaneous oesophageal pressure indicated that the expiratory muscle force never achieved the high values $(>10$ $20 \mathrm{cmH}_{2} \mathrm{O}$ ) required to achieve maximum expiratory flow (fig. 1a). Upper airway dysfunction can cause interruptions in exhalation, but this is usually accompanied by normal or high oesophageal pressures, and thus did not adequately explain our patient's halting exhalations. Static deflation pressurevolume curves of the lung revealed a normally compliant lung (fig. 1b). Oesophageal pressures during maximal inspiratory efforts against an occluded airway are expected to be most negative at low lung volumes, in our patient they were normally negative at high lung volumes, but were progressively less negative at lower lung volumes, suggesting an inability to activate inspiratory muscles at lower volumes (fig. 1b). This pattern is not consistent with simple inspiratory muscle weakness, but rather with an abnormality of respiratory muscle control, which is also suggested by her halting exhalations. Maximal expiratory efforts against an occlusion showed abnormally low oesophageal pressures, $+19 \mathrm{cmH}_{2} \mathrm{O}$ (normal value $>80 \mathrm{cmH}_{2} \mathrm{O}$ ). Ultrasound examination of diaphragmatic motion showed no evidence of the paradoxical movement of the diaphragm during inspiration that would indicate diaphragmatic weakness.

MS is a disease of the central nervous system and consists of acute intermittent episodes of nerve demyelination that stabilise and later partially regress. Demyelination commonly affects the motor pathways, especially of the limbs, resulting in weakness and impaired mobility. Pulmonary complications and infections are another major cause of morbidity in MS, and their attributable mortality is as much as two-fold higher than in the general population [2]. Pulmonary manifestations of MS consist primarily of respiratory muscle weakness that leads to impaired ventilation and poor cough [1, 3-6]. Several reports have suggested that the expiratory muscles are preferentially involved $[1,3]$. The diagnosis of respiratory muscle dysfunction is often made clinically with the aid of standard PFTs. Several studies have shown a consistent decrease in maximal muscle forces in ambulatory asymptomatic patients without additional pulmonary functional abnormalities [1, 4, 5]. There is a correlation between more severe reductions in muscle forces and disease severity [1]. This weakness probably results from progressive demyelination in the descending medullary and cervical upper motor neuron pathways, or involvement of respiratory lower motor neurons in the cervical and thoracic cord [7]. It is also likely to be exacerbated by conditions common in MS and known to lead to impairments in muscle function, such as deconditioning, treatment with corticosteroids, and inflammatory cytokines [1]. Acute onset of respiratory muscle weakness, including isolated bilateral diaphragmatic weakness, has been rarely reported and is associated with acute demyelinating lesions in the medulla or spinal cord. Such acute weakness can remit, as do other neurological manifestation of MS [8].

As demyelination can affect the brainstem, MS could also result in disorders of respiratory control. Ratchet breathing, characterised by jerky breaths with short apnoeic pauses interrupting airflow, has been associated with localised lesions in the medulla [7]. Few studies have investigated respiratory dyscoordination in MS. TANTUCCI et al. [3] studied control of breathing and muscle strength in 11 non-dyspnoeic patients with moderate MS. They reported an increased respiratory drive, as assessed by the $P_{0.1}$ (airway occlusion pressure at $0.1 \mathrm{~s}$ ), at rest and in response to inspired $\mathrm{CO}_{2}$, but with an associated decreased ventilatory response to $\mathrm{CO}_{2}$ consistent with muscle weakness or chest wall factors. The authors could not determine from the P0.1 measurement alone if there were additional defects in coordination. In a case series, HowARD et al. [7] reported six patients with MS and evidence of disordered respiratory control 

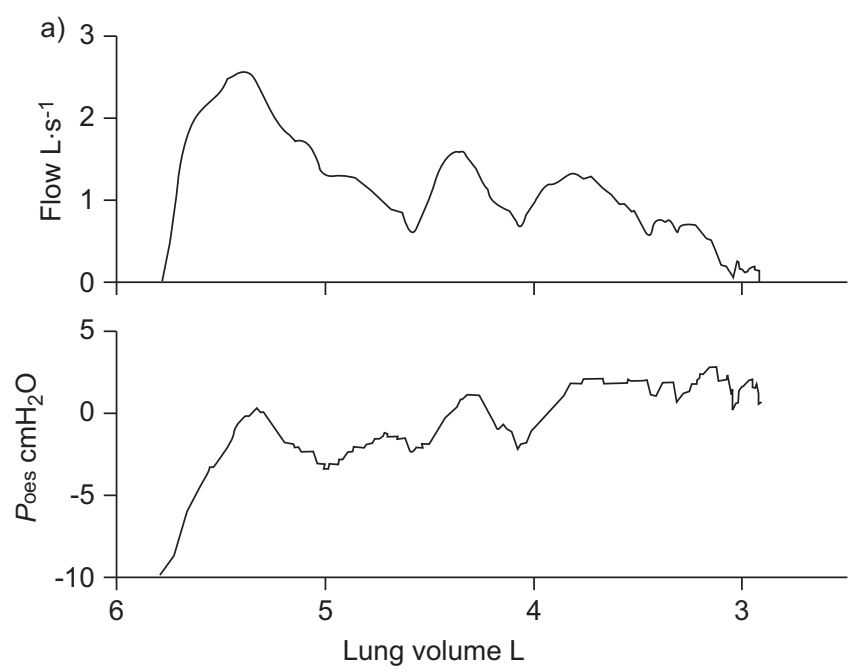

b)

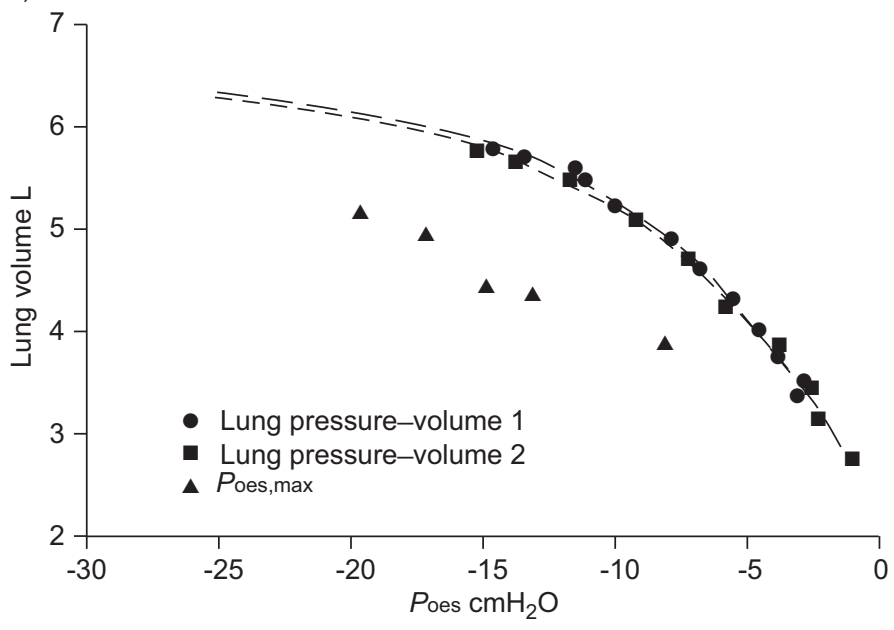

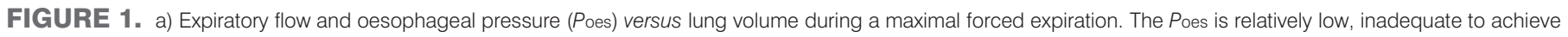

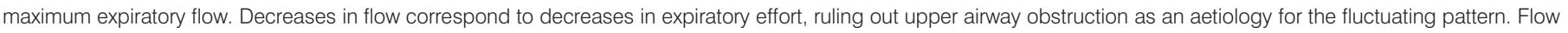

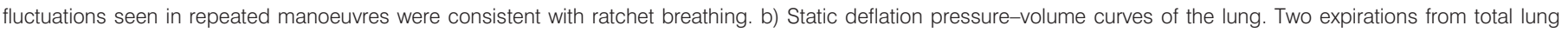

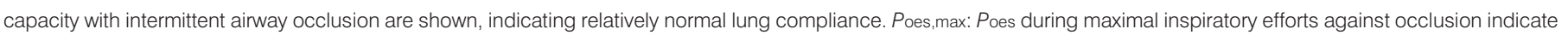

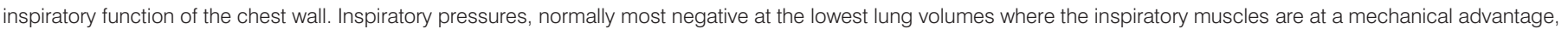
were most negative at higher lung volumes, reaching $-20 \mathrm{cmH}_{2} \mathrm{O}$ (nearly normal).

on physical examination. The patients had either an irregular breathing pattern or an inability to voluntarily control ventilation. For one patient, in whom a post mortem examination was performed, demyelination plaques were evident in the brainstem. All patients had either tetraplegia, previous symptoms of demyelination in the brainstem, or advanced respiratory muscle weakness when they presented.

Oesophageal manometry is a minimally invasive test that enables the measurement of isolated lung or chest wall compliance and respiratory muscle forces. With the additional use of gastric manometry or ultrasound, a diagnosis of bilateral diaphragmatic weakness can be made, a diagnosis that is often missed with fluoroscopy alone [9]. Recent evidence has shown the clinical usefulness of oesophageal manometry in the setting of mechanical ventilation and critical illness [10]. In ambulatory patients, it can be a useful adjunct to PFTs in cases of unexplained dyspnoea, especially when there is suspicion of restrictive chest wall factors or respiratory muscle or diaphragmatic impairment.

This highly functional MS patient developed subacute dyspnoea with restriction on PFTs and a reduction of the vital capacity in the supine position without EMG evidence of myopathy or nerve conduction abnormalities. After performing oesophageal manometry, diaphragmatic weakness was adequately excluded, and the patient was noted to have ratchet exhalation, low expiratory pleural pressures, and, most remarkably, an unusual pattern of a least negative maximal inspiratory pressure at the lowest measured lung volume. The normal EMG, diaphragmatic ultrasound and the unusual pattern of low inspiratory pressures argue against muscle weakness as the aetiology, and support MS-related respiratory muscle dyscoordination as a more likely cause. The abnormally low expiratory pressures at all measured lung volumes suggest a more profound dyscoordination of exhalation. The differential impact on expiratory function and the largely spontaneous improvement in the patient's symptoms also argue for a new MS-associated lesion, presumably in the brainstem, although an MRI could not be performed. We speculate that the supine worsening of her dyspnoea and vital capacity are explained by worsening dyscoordination in this position. To our knowledge this is the first case of impairment of respiratory muscle control in a patient with early-stage MS presenting with dyspnoea and diagnosed by oesophageal manometry.

\section{Maha R. Farhat*, Stephen H. Loring", Peter Riskind and Gerald Weinhouse ${ }^{+}$}

*Division of Pulmonary and Critical Care, Massachusetts General Hospital, Harvard Medical School, Boston, MA, "Dept of Anaesthesia and Critical Care, Beth Israel Deaconess Medical Center, Harvard Medical School, Boston, MA, "Dept of Neurology, University of Massachusetts Memorial Hospital, Worcester, MA, and 'Dept of Pulmonary and Critical Care, Brigham and Women's Hospital, Harvard Medical School, Boston, MA, USA.

Correspondence: M.R. Farhat, Division of Pulmonary and Critical Care, Massachusetts General Hospital, 55 Fruit Street, Bulfinch 148, Boston, MA 02114, USA. E-mail: mrfarhat@partners.org

Support Statement: S.H. Loring was supported by a grant from the National Institutes of Health (HL-52586).

Statement of Interest: None declared.

\section{REFERENCES}

1 Gosselink R, Kovacs L, Decramer M. Respiratory muscle involvement in multiple sclerosis. Eur Respir J 1999; 13: 449-454. 
2 Hurwitz BJ. Analysis of current multiple sclerosis registries. Neurology 2011; 76: Suppl. 1, S7-S13.

3 Tantucci C, Massucci M, Piperno R, et al. Control of breathing and respiratory muscle strength in patients with multiple sclerosis. Chest 1994; 105: 1163-1170.

4 Buyse B, Demedts M, Meekers J, et al. Respiratory dysfunction in multiple sclerosis: a prospective analysis of 60 patients. Eur Respir J 1997; 10: 139-145.

5 Mutluay FK, Gürses HN, Saip S. Effects of multiple sclerosis on respiratory functions. Clin Rehabil 2005; 19: 426-432.

6 Smeltzer SC, Utell MJ, Rudick RA, et al. Pulmonary function and dysfunction in multiple sclerosis. Arch Neurol 1988; 45: 1245-1249.
7 Howard RS, Wiles CM, Hirsch NP, et al. Respiratory involvement in multiple sclerosis. Brain 1992; 115: 479-494.

8 Kuwahira I, Kondo T, Ohta Y, et al. Acute respiratory failure in multiple sclerosis. Chest 1990; 97: 246-248.

9 Benditt JO. Esophageal and gastric pressure measurements. Respir Care 2005; 50: 68-75.

10 Talmor D, Sarge T, Malhotra A, et al. Mechanical ventilation guided by esophageal pressure in acute lung injury. $N$ Engl J Med 2008; 359: 2095-2104.

\section{Multidrug-resistant tuberculosis in UK children: presentation, management and outcome}

\section{To the Editor:}

Multidrug-resistant tuberculosis (MDR-TB), defined as Mycobacterium tuberculosis resistant to at least isoniazid and rifampicin, is an increasing problem globally [1]. Children are not usually included in global surveys of MDR-TB incidence and prevalence [2] due to the diagnostic challenges of paucibacillary TB [3]. Data on the burden of MDR-TB in children are, therefore, lacking globally [1, 4, 5] and no published data from the UK exist. The current World Health Organization (WHO) [6] and National Institute for Clinical Excellence (NICE) [7] TB guidelines for the management of MDR-TB do not explicitly address paediatric disease. As a consequence, the complex management of this condition is left to individual clinicians' judgment. In general, childhood TB is paucibacillary, most cases are treated on clinical grounds without drug susceptibility testing [2], and correct and timely diagnosis of childhood MDR-TB is therefore challenging. There are limited data on the pharmacokinetics, dosing and safety of the many second-line drugs used to treat MDR-TB in children [5]. Our group is the first to review the management of a paediatric cohort with MDR-TB in the UK, and represents the largest available dataset from children with culture-confirmed MDR-TB from western Europe and North America published to date.

We conducted a retrospective audit of children diagnosed with culture confirmed MDR-TB between January 1, 2006 and December 31, 2010 and summarise presentation, management and outcome of this cohort. Data were included if the subjects were under 16 years old and had culture-confirmed MDR-TB reported to the Health Protection Agency (HPA). Additional, fully anonymised data, including HIV status, country of child and parents' birth, radiology, history of previous treatment for TB, symptoms, management, duration of therapy, use of directly observed therapy (DOT), duration of hospital admission and follow-up were obtained. Results of drug susceptibility testing for first- and second-line drugs, using the resistance ratio (proportion) method, were obtained from the UK Mycobacterial Surveillance Network [8, 9]. All second line antibiotic susceptibility testing was carried out by the supra-national laboratory. The period of follow-up was 2 years from the time of presentation.

The following definitions were used. Culture-confirmed MDRTB cases: children who were symptomatic and had cultureconfirmed M. tuberculosis resistant to rifampicin and isoniazid. Favourable treatment outcome: clinical and radiological resolution of symptoms and treatment completion 24 months post commencing MDR-TB treatment, as reported by the treating clinician. Treatment delay: the time from start of presentation to hospital to initiation of MDR-TB treatment.

17 children with culture-confirmed MDR-TB were notified between 2006-2010. The median age was 13 years (range 115 years) and the majority were female (13 out of 17). Details of the cases are presented in table 1.

There were few reported side-effects of treatment. Para-aminosalicylic acid was poorly tolerated by three of the four children who received it; two children had gastrostomy tubes inserted for treatment purposes and one developed hypothyroidism.

Since it was conducted as an audit, a limitation of our study is the retrospective nature of the collected data from multiple centres and HPA with some data gaps. Furthermore, due to the small sample size, caution should be exercised when generalising these results to a wider or future population of MDR-TB paediatric cases.

Nevertheless, although clinical practice showed considerable variability, our cohort seemed to have had favourable outcomes and few side-effects were reported. Most clinicians treating this cohort adhered to the WHO guidelines for programmatic management of drug-resistant tuberculosis, published in 2011 [6], which recommend (based on low quality evidence) the use of four second-line anti-tuberculous drugs that are likely to be effective, including a parenteral agent. However, many of the children were initially started on drugs that were found to be ineffective once drug sensitivity testing data became available. Of the children with available data regarding length of treatment, many received treatment for less than the 20 months recommended for adult patients. 